\title{
Some Features on Entropy Squeezing for Two-Level System with a New Nonlinear Coherent State
}

\author{
Salama I. Ali' ${ }^{1}$, Sayed Abdel-Khalek ${ }^{2}$ \\ ${ }^{1}$ Mathematics Department, Faculty of Science, Al-Azher University, Cairo, Egypt \\ ${ }^{2}$ Mathematics Department, Faculty of Science, Sohag University, Sohag, Egypt \\ Email: salama5laser@yahoo.com
}

Received 13 January 2014; revised 22 February 2014; accepted 12 March 2014

Copyright (C) 2014 by authors and Scientific Research Publishing Inc.

This work is licensed under the Creative Commons Attribution International License (CC BY). http://creativecommons.org/licenses/by/4.0/

(c) (i) Open Access

\begin{abstract}
Entropy squeezing is an important feature in performing different tasks in quantum information processing such as quantum cryptography and superdense coding. These quantum information tasks depend on finding the states in which squeezing can be created. In this article, a new feature on entropy squeezing for a two level system with a class of nonlinear coherent state (NCS) is observed. An interesting result on the comparison between the coherent state (CS) and NCS is explored. The influence of the Lamb-Dick parameter in both absence and presence of the Kerr medium is examined. A rich feature of entropy squeezing in the case of NCS, which is observed to describe the motion of the trapped ion, has been obtained.
\end{abstract}

\section{Keywords}

Entropy Squeezing; Kerr-Like Medium; Nonlinear Coherent State

\section{Introduction}

It is known that quantum entangled state plays an important role in the fields of quantum information theory as well as quantum teleportation and computation. The entropy which automatically includes all moments of the density operator has been shown to be a very useful operational measure of the purity of the quantum state. The most important and interesting work to understand relation between entropy and information was done by Shannon [1], who introduced the entropy (Shannon entropy) into communications theory. Recently it has been shown that nonlinear coherent states are useful in the description of the motion of a trapped ion and various non-classical properties of such states have also been studied [2]. We note that in Refs [2] and [3] nonlinear coherent states 
have been defined as the right eigenstate of a generalized annihilation operator $\hat{A}$ (which emerges from the Hamiltonian describing the dynamics) this is because in the case of nonlinear algebras the commutator $\left[\hat{A}, \hat{A}^{+}\right]$ is not a constant or a linear function of the generators of the algebra but nonlinear in the in the generators. As a consequence it is difficult to obtain an explicit form of nonlinear coherent state constructed via the displacement operator technique. The nonlinear coherent state (NCS) was introduced as a new state of the source of the coherent field to describe some of the non-classical properties like squeezing and Sub-Poissnian behavior [4]. There are some previous studies on the entropy squeezing of a two-level atom, such as one photon transition [5], the nonlinear Kerr medium [6] and degenerate two-photon process [7]. The authors of these papers have focused only on the initial coherent state of the field.

Recently, much attention has been drawn to squeezing in an ensemble of atoms illuminated with light, involving quantum noise and atomic spin polarization measurement [8], and quantum-controlled few-photon states generated by squeezed atoms [9]. These studies of atomic squeezing are based on the Heisenberg uncertainty relation (HUR), which is regarded as the standard limitation on measurements of quantum fluctuations. HUR is formulated in terms of the variances or standard deviations of the system observable. As an alternative to the HUR, Hirschman [10] studied quantum uncertainty by using quantum entropy theory, and obtained an entropic uncertainty relation for position and momentum which can overcome the limitations of the HUR.

The entropy squeezing and variance squeezing for the entangled sate of a single two-level atom interacting with a single electromagnetic field in a squeezed vacuum a broad bandwidth are studied [11]. Also, the similarities and differences of both reservoirs for the two different models have been explained through some calculations, such as the atomic inversion and the von Neumann entropy. It is to be noted that considering a mode structure plays a role like the squeezing parameter in the case of a squeezed vacuum reservoir. Also, the entropy squeezing of a two-level atom driven by a strong classical field and damped into a modeled reservoir with non-flat density of modes has been investigated [12]. On the other hand, the dynamics of a single atom entropy squeezing of the two-qubit system, in the presence of local squeezed reservoirs, has been discussed. Our aim in the present paper is to investigate the entropy squeezing of a two level atom when the initial state of the field is taken to be NCS and discuss different features of entropy squeezing in the case of NCS. These features are connected with the Lamb-Dick parameter. Here we also examine the influence of a nonlinear medium and the detuning parameter on the squeezing parameter of the atomic operators $\hat{S}_{x}, \hat{S}_{y}$ and $\hat{S}_{z}$. The organization of the paper is arranged as follows. In Section 2, we present a brief review of the Hamiltonian model and give an exact expression for the density matrix $\hat{\rho}(t)$. In Section 3, we employ the density matrix to investigate the properties of the entropy squeezing. Finally, we give our discussion in Section 4.

\section{Dynamics of One Photon JCM}

In this paper, we focus our attention to a quantum optical model, where a single two-level atom via one photon process, interacts with a single quantized cavity mode of the radiation field. Then the Hamiltonian of the above system of interest may be written as

$$
\hat{H}=\omega_{f} \hat{a}^{\dagger} \hat{a}+\omega_{A} \hat{S}_{z}+\kappa \hat{a}^{\dagger 2} \hat{a}^{2}+\gamma\left(\hat{a}^{\dagger} \hat{S}_{-}+\hat{a} \hat{S}_{+}\right),
$$

where $\omega_{f}$ is the field frequency, $\omega_{A}$ is the atomic frequency, $\hat{a}$ and $\hat{a}^{?}$ are the annihilation and the creation operators for the mode of the cavity field satisfying $\left[\hat{a}, \hat{a}^{\dagger}\right]=1$ and $\hat{S}_{ \pm}$and $\hat{S}_{z}$ are the atomic spin operators defined by

$$
\left.\begin{array}{l}
\hat{S}_{z}=\frac{1}{2}(|\uparrow\rangle\langle\uparrow|-| \downarrow\rangle\langle\downarrow|) \\
\left.\hat{S}_{+}=|\uparrow\rangle\langle\downarrow| \text { and } \hat{S}_{-}=|\downarrow\rangle\langle\uparrow|\right)
\end{array}\right)
$$

We denoted by $|\uparrow\rangle$ and $|\downarrow\rangle$ the upper and lower states of the atom, respectively and $\gamma$ is the effective coupling constant. Also, we denoted by $\kappa$ the dispersive part of the third-order nonlinearity of the Kerr-like medium, with the detuning parameter $\Delta=\omega_{A}-\omega_{f}$. Therefore, we employ the unites of $\hbar=1$. The effective Hamiltonian can be written as

$$
\hat{H}_{\text {eff }}=\hat{H}_{0}+\hat{H}_{I}
$$

where, 


$$
\left.\begin{array}{l}
\hat{H}_{0}=\omega_{f}\left(\hat{a}^{\dagger} \hat{a}+\hat{S}_{z}\right), \\
\hat{H}_{I}=\gamma \Delta \hat{S}_{z}+\gamma \kappa \hat{a}^{\dagger 2} \hat{a}^{2}+\gamma\left(\hat{a}^{\dagger} \hat{S}_{-}+\hat{a} \hat{S}_{+}\right)
\end{array}\right)
$$

For convenience, we take, $\chi=\gamma \kappa, \delta=\gamma \Delta$ and assume that the atom is initially in the superposition state $\left|\psi_{A}(0)\right\rangle=A_{\uparrow}|\uparrow\rangle+A_{\downarrow}|\downarrow\rangle$. Also, we assume that the field is initially in the new nonlinear coherent state (NCS),

$$
|\alpha\rangle=\sum_{n=0}^{\infty} B(n)|n\rangle
$$

where, $B(n)$ is the distribution function of the NCS. The NCS is defined as $|\alpha\rangle_{d}=\sum_{n=0}^{\infty} D(n)|0\rangle$ i.e., it is a coherent state (CS) corresponding to the second algebra [13]. One can write $|\alpha\rangle$ in the following form [4]-[7] [13]

$$
|\alpha\rangle_{d}=N \sum_{n=0}^{\infty} \frac{\alpha^{n} d_{n}}{\sqrt{n !}}|n\rangle
$$

where $N$ is a normalization constant, which can be determined from the condition $\langle\alpha \mid \alpha\rangle_{d}=1$ and is given by

$$
N^{2}=\left\{\sum_{n=0}^{\infty} \frac{d_{n}^{2}}{n !}\left(\alpha^{*} \alpha\right)^{n}\right\}^{-1} .
$$

While $d_{0}=1$ and the coefficients $d_{n}=\{f(n) !\}^{-1}$, it is clear that for different choices of the nonlinearity function, we shall get different nonlinear coherent states. In the present case we choose a nonlinearity function, which has been used in the description of the motion of a trapped ion [14].

$$
f(n)=L_{n}^{1}\left(\eta^{2}\right)\left[(n+1) L_{n}^{0}\left(\eta^{2}\right)\right]^{-1}
$$

where $\eta$ is known as the Lamb-Dick parameter and $L_{n}^{m}(x)$ are generalized Lagurre polynomials given by

$$
L_{n}^{m}\left(\eta^{2}\right)=\sum_{i=0}^{\infty}\left(\begin{array}{c}
m+i \\
n+i
\end{array}\right) \frac{\left(-\eta^{2}\right)^{i}}{i !}
$$

Clearly $f(n)=1$ when $\eta=0$ and in this case nonlinearity coherent states become the standard coherent states. However, when $\eta \neq 1$ nonlinearity starts developing with degree of depending on the magnitude of $\eta$ [14]. Then the initial state of the atom-field coupling system reads as

$$
|\psi(0)\rangle=\sum_{n=0}^{\infty} F(n)\left\{A_{\uparrow}|n, \uparrow\rangle+A_{\downarrow}|n, \downarrow\rangle\right\}
$$

where, $\sum_{n=0}^{\infty}|B(n)|^{2}=1, A_{\uparrow}=\cos (\theta / 2)$ and $A_{\downarrow}=\sin (\theta / 2) \exp (-\mathrm{i} \phi)$. Here $\theta \in[0, \pi]$ denotes the initial coherence of the two-level atom and $\varphi \in[0,2 \pi]$ is the relative phase between the upper and lower states of the two-level atom. Thus the initial density operator of the system is given by $\rho(0)=\rho_{A}(0) \otimes \rho(0)_{F}$ where, $\rho_{F}(0)=|\alpha\rangle\langle\alpha|$ and $\rho_{A}(0)=\left|\psi_{A}(0)\right\rangle\left\langle\psi_{A}(0)\right|, \rho(0)$ describes the initial values for the field-atom density operator.

At any time $t>0$ the solution of the Schrödinger equation

$$
\mathrm{i} \frac{\mathrm{d}|\psi(t)\rangle}{\mathrm{d} t}=H_{I}|\psi(t)\rangle
$$

for the state vector $|\psi(t)\rangle$ with the initial condition (6) is

$$
\left.|\psi(t)\rangle=\sum_{n=0}^{\infty} B(n)\left\{\psi_{\uparrow}(n, t)|n, \uparrow\rangle+\psi_{\downarrow}(n, t) \mid n, \downarrow\right)\right\}
$$


and the density matrix of system is

$$
\begin{aligned}
\rho(t)= & \sum_{n=0}^{\infty} \sum_{m=0}^{\infty} \psi_{\uparrow}(n, t) \psi_{\uparrow}^{*}(m, t)|n, \uparrow\rangle\left\langle\uparrow, m\left|+\psi_{\uparrow}(n, t) \psi_{\downarrow}^{*}(m, t)\right| n, \uparrow\right\rangle\langle\uparrow, m| \\
& +\psi_{\downarrow}(n, t) \psi_{\uparrow}^{*}(m, t)|n, \uparrow\rangle\left\langle\uparrow, m\left|+\psi_{\downarrow}(n, t) \psi_{\downarrow}^{*}(m, t)\right| \mid n, \uparrow\right\rangle\langle\uparrow, m|
\end{aligned}
$$

where the coefficient $\psi_{\uparrow}(n, t)$ and $\psi_{\downarrow}(n, t)$ are given by

$$
\begin{gathered}
\psi_{\uparrow}(n, t)=\exp \left(-\mathrm{i} \gamma t \chi n^{2}\right)\left\{A_{\uparrow} B(n)\left[\cos \Upsilon_{n}-\mathrm{i}\left(\frac{\Delta}{2}-\chi n\right) \frac{\sin \Upsilon_{n}}{\Omega_{n}}\right]-\mathrm{i} A_{\downarrow} \sqrt{n+1} B(n+1) \frac{\sin \Upsilon_{n}}{\Omega_{n}}\right\} \\
\psi_{\downarrow}(n, t)=\exp \left[-\mathrm{i} \gamma t \chi(n-1)^{2}\right]\left\{A_{\downarrow} B(n)\left[\cos \Upsilon_{n-1}+\mathrm{i}\left(\frac{\Delta}{2}-\chi(n-1)\right) \frac{\sin \Upsilon_{n-1}}{\Omega_{n-1}}\right]-\mathrm{i} A_{\uparrow} \sqrt{n} B(n-1) \frac{\sin \Upsilon_{n}-1}{\Omega_{n-1}}\right\}
\end{gathered}
$$

where, $\Upsilon_{n}=\gamma t \Omega_{n}, \Omega_{n}=\sqrt{\left(\frac{\Delta}{2}-\chi n\right)^{2}+n+1}$ with $\Omega_{n}$ is the Rabi frequency which depends on the detuning parameter and nonlinear medium parameter.

\section{Atomic Inversion}

We mainly devote the present section to considering the atomic inversion, from which the phenomenon of collapses and revivals can be observed. However, we shall first introduce some expressions for the probability amplitude. The expressions $\left|\psi_{\uparrow}(n, t)\right|^{2}$ and $\left|\psi_{\downarrow}(n, t)\right|^{2}$ represent the probabilities that at time $t$, the field has $n$ photons present and the atom is in level $|\uparrow\rangle$ and $|\downarrow\rangle$ respectively. The probability $P(n, t)$ that there is $n$ photons in the field at time $t$ is therefore obtained by taking the trace over the atomic states, i.e.,

$$
P(n, t)=\left|\psi_{\uparrow}(n, t)\right|^{2}+\left|\psi_{\downarrow}(n, t)\right|^{2}
$$

where $P(n, 0)$ is the probability that there are $n$ photons present the field at time $t=0$, which is given for a NCS for the field by

$$
P(n, 0)=|B(n)|^{2}
$$

Another important quantity one may consider is the atomic inversion $W(t)$, which is related to the probability amplitudes $\left|\psi_{\uparrow}(n, t)\right|^{2}$ and $\left|\psi_{\downarrow}(n, t)\right|^{2}$ by the expression

$$
W(t)=\frac{1}{2} \sum_{n=0}^{\infty}\left|\psi_{\uparrow}(n, t)\right|^{2}-\left|\psi_{\downarrow}(n, t)\right|^{2}
$$

Thus from Equation (13) and after some rearrangements, we can obtain

$$
\begin{aligned}
& W(t)=\frac{1}{2} \sum_{n=0}^{\infty}|B(n)|^{2}\left\{\cos ^{2}\left(\frac{\theta}{2}\right)\left[\cos ^{2} \Upsilon_{n}+\left(\frac{\Delta}{2}-\chi n\right)^{2} \frac{\sin ^{2} \Upsilon_{n}}{\Omega_{n}^{2}}\right]-\sin ^{2}\left(\frac{\theta}{2}\right)\left[\cos ^{2} \Upsilon_{n-1}+\left(\frac{\Delta}{2}-\chi(n-1)\right)^{2} \frac{\sin ^{2} \Upsilon_{n-1}}{\Omega_{n-1}^{2}}\right]\right. \\
& +\frac{1}{2} \sum_{n=0}^{\infty}\left\{|B(n+1)|^{2}\left(\frac{\gamma \sqrt{n+1} \sin \Upsilon_{n+1} \sin \left(\frac{\theta}{2}\right)}{\Omega_{n}}\right)-|B(n-1)|^{2} \times\left(\mid \frac{\gamma \sqrt{n} \sin \Upsilon_{n-1} \cos \left(\frac{\theta}{2}\right)}{\Omega_{n}-1}\right) \mid\right\} \\
& -\frac{\sin \theta}{2} \sum_{n=0}^{\infty}\left(|B(n) B(n+1)| \gamma \sqrt{n+1} \frac{\sin \Upsilon_{n}}{2 \Omega_{n}}-|B(n) B(n-1)| \frac{\sqrt{n} \sin \Upsilon_{n-1}}{2 \Omega_{n-1}}\right) \sin \phi-(|B(n) B(n+1)| \gamma \sqrt{n+1} \\
& \left.[\delta-\chi n] \frac{\sin ^{2} \Upsilon_{n}}{\Omega_{n}^{2}}-\sqrt{n}|B(n) B(n-1)|\left[\frac{\Delta}{2}-\chi(n-1)\right] \frac{\sin ^{2} \Upsilon_{n-1}}{\Omega_{n-1}^{2}}\right) \cos \phi
\end{aligned}
$$




\section{Entropy Squeezing}

In this paper, we use the Heisenberg uncertainty relation (HUR) to study the squeezing information entropy. It has been pointed out that the Heisenberg uncertainty relation (HUR) cannot give sufficient information on the atomic squeezing in some cases [13]. For instance, for a two-level atom, characterized by Pauli operator $\hat{S}_{x}, \hat{S}_{y}$ and $\hat{S}_{z}$, the uncertainty relation is given by

$$
\Delta \hat{S}_{x} \Delta \hat{S}_{y} \geq \frac{1}{2}\left|\left\langle\hat{S}_{z}\right\rangle\right|
$$

where the Pauli operators $\hat{S}_{x}, \hat{S}_{y}$ and $\hat{S}_{z}$, satisfying the commutation $\left[\hat{S}_{x}, \hat{S}_{y}\right]=\mathrm{i} \hat{S}_{z}$ and $\Delta \hat{S}_{\alpha}=\sqrt{\left\langle\hat{S}_{\alpha}^{2}\right\rangle-\left\langle\hat{S}_{\alpha}\right\rangle^{2}}$. In this way, the fluctuation in the component $\hat{S}_{\alpha}$ of the atomic dipole is said to be squeezed if $\hat{S}_{\alpha}$ satisfies the condition

$$
V\left(\hat{S}_{\alpha}\right)=\left(\Delta \hat{S}_{\alpha}-\sqrt{\left|\frac{\left\langle\hat{S}_{z}\right\rangle}{2}\right|}\right)<0, \quad \alpha=x \text { or } y
$$

An optimal entropic uncertainty relation for sets of $N+1$ complementary observables with non-degenerate eigenvalues in an even $\mathrm{N}$-dimensional Hilbert space has been recently investigated using quantum entropy theory [5]. It takes the form

$$
\sum_{\alpha=1}^{N+1} H\left(\hat{S}_{\alpha}\right) \geq \frac{N}{2} \ln \left(\frac{N}{2}\right)+\left(1+\frac{N}{2}\right) \ln \left(1+\frac{N}{2}\right)
$$

where $H\left(\hat{S}_{\alpha}\right)$ represents the information entropy of the variable $\hat{S}_{\alpha}$. The aim of this paper is to use entropy uncertainty relation EUR (16) as a general criterion for the squeezing in terms of information entropy for a two-level atom in the Jaynes-Cumming model with one-photon process in a non-linear Kerr medium.

The probability distribution for $N$ possible outcomes of measurements for an arbitrary quantum state of an operators $\hat{S}_{\alpha}$ is $P_{i}\left(\hat{S}_{\alpha}\right)=\left\langle\Psi_{\alpha i}|\rho| \Psi_{\alpha i}\right\rangle$, where $\left|\Psi_{\alpha i}\right\rangle$ is an eigenvector of the operator $\hat{S}_{\alpha}$ such that $\hat{S}_{\alpha}\left|\Psi_{\alpha i}\right\rangle=\lambda_{\alpha i}\left|\Psi_{\alpha i}\right\rangle, \alpha=x, y, z, i=1,2, \cdots, N$. The corresponding Shannon information entropies are then defined as

$$
H\left(\hat{S}_{\alpha}\right)=-\sum_{i=1}^{N} P_{i}\left(\hat{S}_{\alpha}\right) \ln P_{i}\left(\hat{S}_{\alpha}\right), \alpha=x, y, z
$$

To obtain the Shannon information entropies of the atomic operators $\hat{S}_{x}, \hat{S}_{y}$ and $\hat{S}_{z}$ for a two-level atom, with $N=2$, one can use the reduced atomic density operator $\hat{\rho}(t)$, thus we have the following expression,

$$
H\left(\hat{S}_{\alpha}\right)=-\frac{1}{2}\left[\rho_{\alpha}(t)+1\right] \ln \left[\frac{1}{2}\left[\rho_{\alpha}(t)+1\right]\right]-\frac{1}{2}\left[1-\rho_{\alpha}(t)\right] \ln \left[\frac{1}{2}\left[1-\rho_{\alpha}(t)\right]\right], \forall \alpha=x, y, z
$$

so that

$$
\begin{gathered}
H\left(\hat{S}_{x}\right)=-\frac{1}{2}\left[\operatorname{Re}\left(\rho_{\downarrow \uparrow}(t)\right)+1\right] \ln \left[\frac{1}{2}\left[\operatorname{Re}\left(\rho_{\downarrow \uparrow}(t)\right)+1\right]\right]-\frac{1}{2}\left[1-\operatorname{Re}\left(\rho_{\downarrow \uparrow}(t)\right)\right] \ln \left[\frac{1}{2}\left[1-\operatorname{Re}\left(\rho_{\downarrow \uparrow}(t)\right)\right]\right], \\
H\left(\hat{S}_{y}\right)=-\frac{1}{2}\left[\operatorname{Im}\left(\rho_{\downarrow \uparrow}(t)\right)+1\right] \ln \left[\frac{1}{2}\left[\operatorname{Im}\left(\rho_{\downarrow \uparrow}(t)\right)+1\right]\right]-\frac{1}{2}\left[1-\operatorname{Im}\left(\rho_{\downarrow \uparrow}(t)\right)\right] \ln \left[\frac{1}{2}\left[1-\operatorname{Im}\left(\rho_{\downarrow \uparrow}(t)\right)\right]\right], \\
H\left(\hat{S}_{z}\right)=-\rho_{\uparrow \uparrow}(t) \ln \left(\rho_{\uparrow \uparrow}(t)\right)-\rho_{\downarrow \downarrow}(t) \ln \left(\rho_{\downarrow \downarrow}(t)\right),
\end{gathered}
$$

where $\rho_{\uparrow \uparrow}(t), \rho_{\downarrow \downarrow}(t), \operatorname{Re} \rho_{\downarrow \uparrow}(t)$ and $\operatorname{Im}\left(\rho_{\downarrow \uparrow}(t)\right)$ are given by 


$$
\begin{aligned}
& \left.\rho_{\uparrow \uparrow}(t)=\cos ^{2}\left(\frac{\theta}{2}\right) \sum_{n=0}^{\infty}\left\{|B(n)|^{2}\left[\cos ^{2} \Upsilon_{n}+\left(\frac{\Delta}{2}-\chi n\right)^{2} \frac{\sin ^{2} \Upsilon_{n}}{\Omega_{n}^{2}}\right]+\sin ^{2}\left(\frac{\theta}{2}\right) \sum_{n=0}^{\infty}|B(n+1)|^{2}\left(\frac{\sqrt{n+1} \sin \Upsilon_{n}}{\Omega_{n}}\right)^{2}\right]\right\} \\
& -\sin (\theta) \sum_{n=0}^{\infty}\left\{\mid B(n) B(n+1)\left(\frac{\sqrt{n+1} \sin \Upsilon_{n}}{\Omega_{n}}\left(\cos \Upsilon_{n} \sin (\phi)-\left[\frac{\Delta}{2}-\chi n\right] \frac{\sin \Upsilon_{n}}{\Omega_{n}} \cos (\phi)\right)\right.\right. \\
& \left.-|B(n-1)|^{2}\left(\frac{\gamma \sqrt{n} \sin \Upsilon_{n-1} \cos \left(\frac{\theta}{2}\right)}{\Omega_{n}-1}\right)^{2}\right\} \\
& \rho_{\downarrow \downarrow}(t)=\sin ^{2}\left(\frac{\theta}{2}\right) \sum_{n=0}^{\infty}|B(n)|^{2}\left(\cos ^{2} \Upsilon_{n-1}+\left[\frac{\Delta}{2}-\chi(n-1)\right] \frac{\sin ^{2} \Upsilon_{n-1}}{\Omega_{n-1}^{2}}\right)+\cos ^{2}\left(\frac{\theta}{2}\right) \sum_{n=0}^{\infty}\left\{|B(n-1)|^{2}\left(\frac{\sqrt{n} \sin \Upsilon_{n-1}}{\Omega_{n-1}}\right)^{2}\right\} \\
& +\sin (\theta) \sum_{n=0}^{\infty}\{|B(n) B(n-1)|\}\left\{\frac{\sqrt{n} \sin \Upsilon_{n-1}}{\Omega_{n-1}}\left(\cos \Upsilon_{n-1} \sin (\phi)-\left[\frac{\Delta}{2}-\chi(n-1)\right] \frac{\sin \Upsilon_{n-1}}{\Omega_{n-1}} \cos (\phi)\right)\right\} \\
& \operatorname{Re} \rho_{\downarrow \uparrow}(t)=\sum_{n=0}^{\infty}\left\{U_{R}(n, t) \cos \left[\gamma t\left(R_{n}-R_{n-1}\right)\right]+U_{v}(n, t) \sin \left[\gamma t\left(R_{n}-R_{n-1}\right)\right]\right\} \\
& \operatorname{Im} \rho_{\downarrow \uparrow}(t)=\sum_{n=0}^{\infty}\left\{U_{R}(n, t) \sin \left[\gamma t\left(R_{n}-R_{n-1}\right)\right]-U_{v}(n, t) \cos \left[\gamma t\left(R_{n}-R_{n-1}\right)\right]\right\} \\
& U_{R}(n, t)=\frac{\sin \theta}{2}\left(X_{n} \cos \phi+Y_{n} \sin \phi|B(n)|^{2}\right)+|B(n+1) B(n-1)| \frac{\sqrt{n(n+1)} \sin \Upsilon_{n} \sin \Upsilon_{n-1} \cos \phi}{\Omega_{n} \Omega_{n-1}} \\
& +|B(n) B(n-1)| \frac{\sqrt{n} \delta_{n} \sin \Upsilon_{n} \sin \Upsilon_{n-1} \cos ^{2}\left(\frac{\theta}{2}\right)}{\Omega_{n} \Omega_{n-1}}-|B(n) B(n+1)| \frac{\sqrt{n+1} \delta_{n-1} \sin \Upsilon_{n} \sin \Upsilon_{n-1} \sin ^{2}\left(\frac{\theta}{2}\right)}{\Omega_{n} \Omega_{n-1}} \\
& U_{V}(n, t)=\frac{\sin \theta}{2}\left(X_{n} \sin \phi-Y_{n} \cos \phi|B(n)|^{2}\right)-|B(n+1) B(n-1)| \frac{\sqrt{n(n+1)} \sin \Upsilon_{n} \sin \Upsilon_{n-1} \sin \phi}{\Omega_{n} \Omega_{n-1}} \\
& +|B(n) B(n-1)| \frac{\sqrt{n} \sin \Upsilon_{n-1} \cos \Upsilon_{n} \cos ^{2}\left(\frac{\theta}{2}\right)}{\Omega_{n-1}}-|B(n) B(n+1)| \frac{\sqrt{n+1} \sin \Upsilon_{n} \sin \Upsilon_{n-1} \sin ^{2}\left(\frac{\theta}{2}\right)}{\Omega_{n}} \\
& X_{n}=\cos \Upsilon_{n} \cos \Upsilon_{n-1}-\frac{\delta_{n} \delta_{n-1} \sin \Upsilon_{n} \sin \Upsilon_{n-1}}{\Omega_{n} \Omega_{n-1}} Y_{n}=\frac{\delta_{n} \sin \Upsilon_{n} \cos \Upsilon_{n-1}}{\Omega_{n}}+\frac{\delta_{n-1} \sin \Upsilon_{n-1} \cos \Upsilon_{n}}{\Omega_{n-1}}
\end{aligned}
$$

Since the uncertainty relation of the entropy can be used as a general criterion for the squeezing of an atom, therefore for a two-level atom. For $N=2$, we have $0 \leq H\left(\hat{S}_{\alpha}\right) \leq \ln 2$, and the Shannon information entropies of the operators $\hat{S}_{x}, \hat{S}_{y}, \hat{S}_{z}$ will satisfy the inequality

$$
H\left(\hat{S}_{x}\right)+H\left(\hat{S}_{y}\right)+H\left(\hat{S}_{z}\right) \geq 2 \ln 2 .
$$

In other words, if we define $\delta H\left(\hat{S}_{\alpha}\right)=\exp \left[H\left(\hat{S}_{\alpha}\right)\right]$, then we can write

$$
\delta H\left(\hat{S}_{x}\right) \delta H\left(\hat{S}_{y}\right) \delta H\left(\hat{S}_{z}\right) \geq 4 .
$$

It is evident that $\delta H\left(\hat{S}_{\alpha}\right)=1$ corresponds to the atom being in a pure state and $\delta H\left(\hat{S}_{\alpha}\right)=2$ corresponds to the atom being in a mixed state. The EUR (16) shows the impossibility of simultaneously having complete information about the observables $\hat{S}_{x}(t), \hat{S}_{y}(t)$ and $\hat{S}_{z}(t)$, where $\delta H\left(\hat{S}_{x}\right)$ and $\delta H\left(\hat{S}_{y}\right)$ respectively measure 
the uncertainties of the polarization components $\hat{S}_{x}$ and $\hat{S}_{y}$.

Now, we define the squeezing of the atom using EUR (16), named squeezing entropy [5]. The fluctuation of the component $\hat{S}_{\alpha}(\alpha=x$ or $y)$ of the atom dipole are said to be "squeezed in entropy" if the information entropy $H\left(\hat{S}_{\alpha}(t)\right)$ of $\hat{S}_{\alpha}$ satisfies the condition

$$
E\left(\hat{S}_{\alpha}\right)=\delta H\left(\hat{S}_{\alpha}\right)-\frac{2}{\sqrt{\delta H\left(\hat{S}_{z}\right)}}<0, \alpha=x, y
$$

$n$ what follows we shall consider the effect of detuning parameter and the nonlinear Kerr like medium on the dynamical behavior of the squeezing entropy of the system under consideration.

\section{Numerical Computation}

On the basis of the analytical solution presented in the previous section, we shall study numerically the dependence of the $E\left(\hat{S}_{x}\right), E\left(\hat{S}_{y}\right)$ entropy squeezing and the atomic inversion $W(t)$, for various parameters of the one photon model. We recall that time $t$ has been scaled; one unit of time is given by the inverse of the coupling constant $\lambda$. In all our plots we take $\eta$ to represent the Lamb-Dicke parameter and the parameter $|\alpha|$. For the case of $\Delta=\chi=0$ (Figure 1), we investigate the influence of the mean photon number on the entropy squeezing
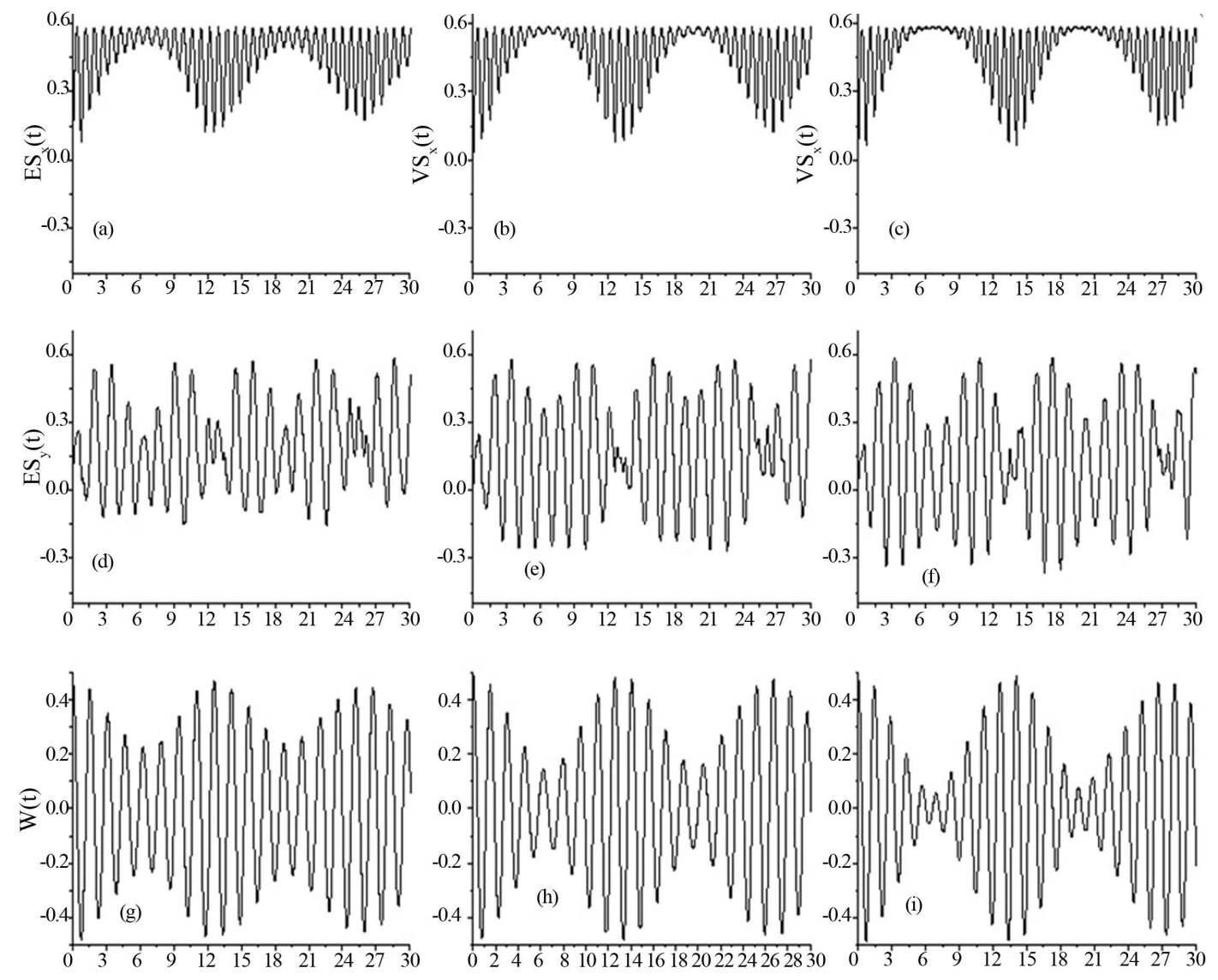

scaled time

Figure 1. The time evolution of the entropy squeezing factors $E\left(\hat{S}_{x}\right), E\left(\hat{S}_{y}\right)$ and population inversion $W(t)$ of a two-level atom interacting with a single-mode in the excited state $\theta=\phi=0$, for parameter $\chi=\Delta=0, \eta=0.8$ and with different values of the parameter $|\alpha|$ where $|\alpha|=1$ in Figures (a,d,g), $|\alpha|=3$ in Figures (b,e,h) and $|\alpha|=6$ in Figures (c,f,i). 
factor $E\left(\hat{S}_{x}\right), E\left(\hat{S}_{y}\right)$, and population inversion $W(t)$. We notice that no squeezing occurs on the atomic variable $\hat{S}_{x}$ as the parameter increased (see Figures 1(a)-(c)).

But Figures 1(d)-(f), present that with increasing the parameter $|\alpha|$, the entropy squeezing on the atomic variables $S_{y}$ is increased. Also the quantum revival is increased but the collapse phenomenon is decreased as $|\alpha|$ increase (see Figures 1 (g)-(i)). These results agree with the case of coherent field but the difference between the coherent state and the nonlinear coherent state appear on the collapse and revival phenomena. For fixed values of the detuning $\Delta$, the detuning parameter and the case of absence the nonlinear medium parameter $\chi=0$, the entropy squeezing factor $E\left(\hat{S}_{x}\right)$ and $E\left(\hat{S}_{y}\right)$ are plotted as a function of the scaled time $t$, where we set three different values of $\Delta$, i.e. $\Delta=1,5,10$. We noticed no entropy squeezing on the atomic variable $\hat{S}_{x}$ as the detuning parameter increases; see Figures 2(a), (c). Although there is great entropy squeezing on when $\Delta=1$, see Figure 2(d) and no squeezing on $\hat{S}_{y}$ when $\Delta=5,10$ see Figures 2(c) and (f).

This is because $W(t)>0$, then

$$
E\left(\hat{S}_{y}\right)=\mathrm{e}^{H\left(\hat{S}_{y}\right)}-2 \mathrm{e}^{-H\left(\hat{S}_{x}\right)}
$$

where $W(t)=\left\langle\hat{S}_{z}\right\rangle$. Also one can see as the detuning parameter increased the period of evolution increased. Figure 3 explains the effect of the detuning parameter when the atom is initially in the superposition state of the upper and lower atomic states and all the other parameters are the same as Figure 2. It is observed that the situation is completely different between the excited and superposition states (see Figures 2 and 3) First we see that when the detuning increases, the entropy squeezing on the atomic variables $\hat{S}_{x}$ increases.

Also there is a great entropy squeezing on the $\hat{S}_{y}$.

In order to see how the entropy squeezing influenced by the nonlinear medium parameter, we set two different values of the Kerr-medium parameter. Figures 4(a), (d) and (g) show the absence of the nonlinear parameter but
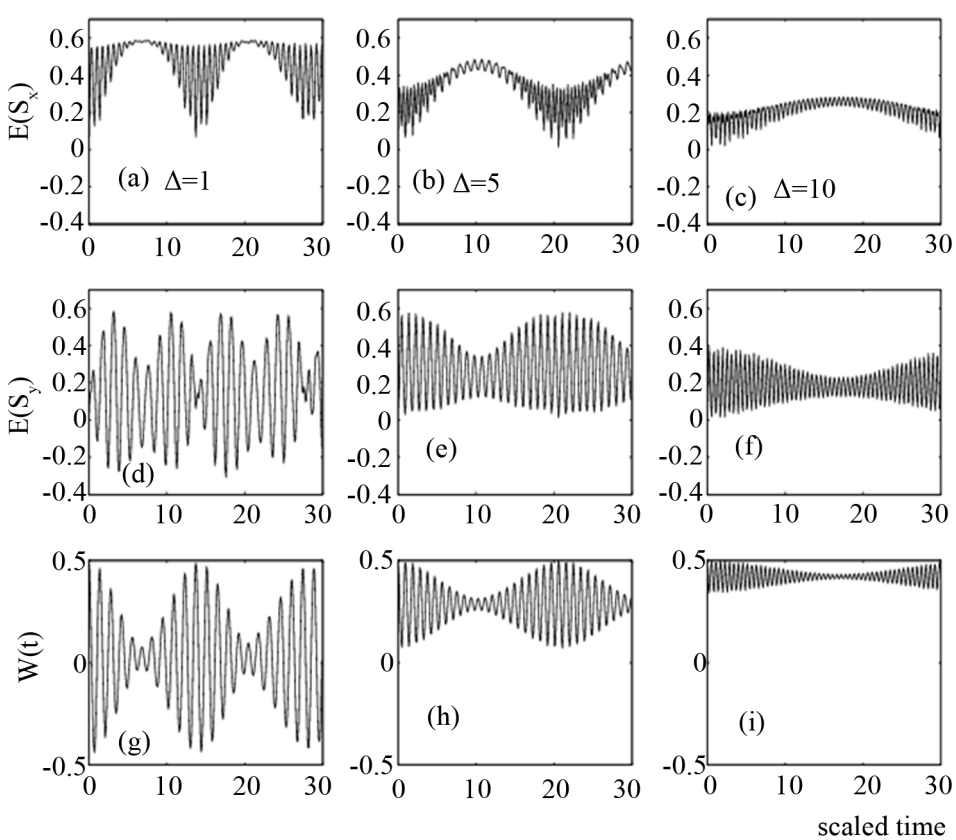

Figure 2. The time evolution of the entropy squeezing factors $E\left(\hat{S}_{x}\right)$, $E\left(\hat{S}_{y}\right)$ and population inversion $W(t)$ of a two-level atom interacting with a single-mode in the excited state $\theta=\varphi=0$, for parameter $\chi=0$, $|\alpha|=6, \eta=0.8$ and with different values of the parameter $\Delta$ where $\Delta=1$ in Figures 2(a) (d) (g), $\Delta=5$ in Figures 2(b) (e) (h) and $\Delta=10$ in Figures 2(c) (f) (i). 

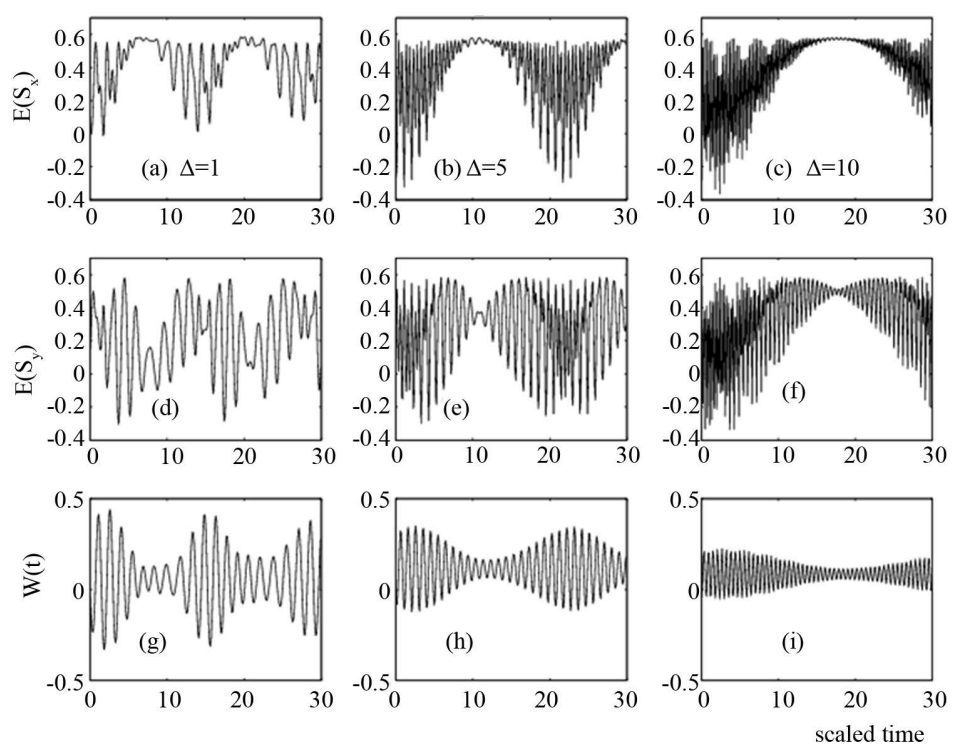

Figure 3. The time evolution of the entropy squeezing factors $E\left(\hat{S}_{x}\right), E\left(\hat{S}_{y}\right)$ and population inversion $W(t)$ of a two-level atom interacting with a single-mode as Figure 2 but $\theta=\pi / 2$ and $\phi=\pi / 4$.
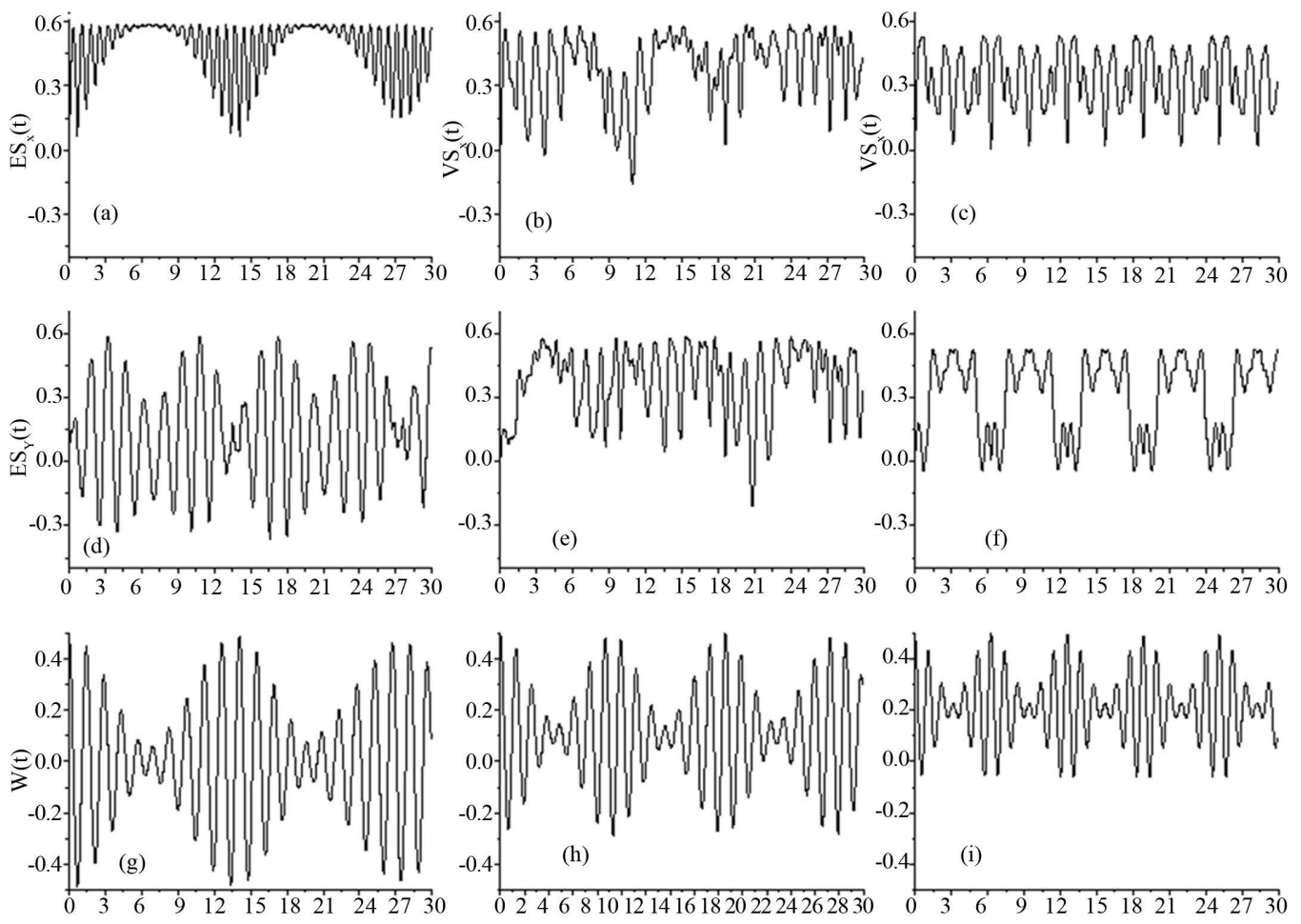

scaled time

Figure 4. The time evolution of the entropy squeezing factors $E\left(\hat{S}_{x}\right), E\left(\hat{S}_{y}\right)$ and population inversion $W(t)$ of a two-level atom interacting with a single-mode in the excited state $\theta=\varphi=0$, for parameter $|\alpha|=6, \Delta=0, \eta=0.6$ and with different values of the Kerr nonlinearity parameter $\chi$ where $\chi=0$ in Figures 4(a) (d) (g), $\chi=0.3$ in Figures 4(b) (e) (h) and $\chi=0.5$ in Figures 4(c) (f) (i). 
the other figures show the influence of the nonlinear parameter. It is remarkable that the nonlinear parameter leads to the following effects, no entropy squeezing occurs on $\hat{S}_{x}$ when $\chi=0$, but there is entropy squeezing on $\hat{S}_{y}$. When $\chi=0.3$, there is an optimal entropy squeezing on both the atomic variables $\hat{S}_{x}$ and $\hat{S}_{y}$. For large values of $\chi$ as $\chi=0.5$ one can see there are more and more optimal entropy squeezing on both of the atomic variables $\hat{S}_{x}$ and $\hat{S}_{y}$.

Also the entropy squeezing factors $E\left(\hat{S}_{x}\right)$ and $E\left(\hat{S}_{y}\right)$ are periodic functions, which have a period $\pi$ and $2 \pi$ respectively (see Figures 4 (c) and (f)).

In order to explain the difference between the sources of the radiation fields of the coherent state and the nonlinear coherent state, we must set different values of the Lamb-Diche parameter $\eta(0,0.5,0.9)$. When $\eta=0$, then the field is initially in the coherent state, but when $\eta \neq 0$ the field is initially in the nonlinear coherent state. Figures 5(a), (d) and (g) show the entropy squeezing in the case of coherent state, which has been examined in many papers [2] [5] [7]. But Figures 5(b), (e) and (h) show the difference between the coherent and new nonlinear coherent states, where we set $\eta=0.5$ while we set $\eta=0.9$ in Figures 5(d), (f) and (i). One can see there is strong entropy squeezing on the atomic variables $\hat{S}_{y}$ and there is an entropy squeezing and the period of collapse also increases.

\section{Conclusions}

We have treated the entropy squeezing of a two-level atom when the field is initially prepared in the NCS. New results can be explored as follows.

1) When the field is initially in an NCS, then rich features of the entropy squeezing can be observed, then the entropy squeezing is a good measurement of the information concerning the case of trapped ion.

2) There is a great difference between the influence of the nonlinearity function $f(n)$, which is used in the de-
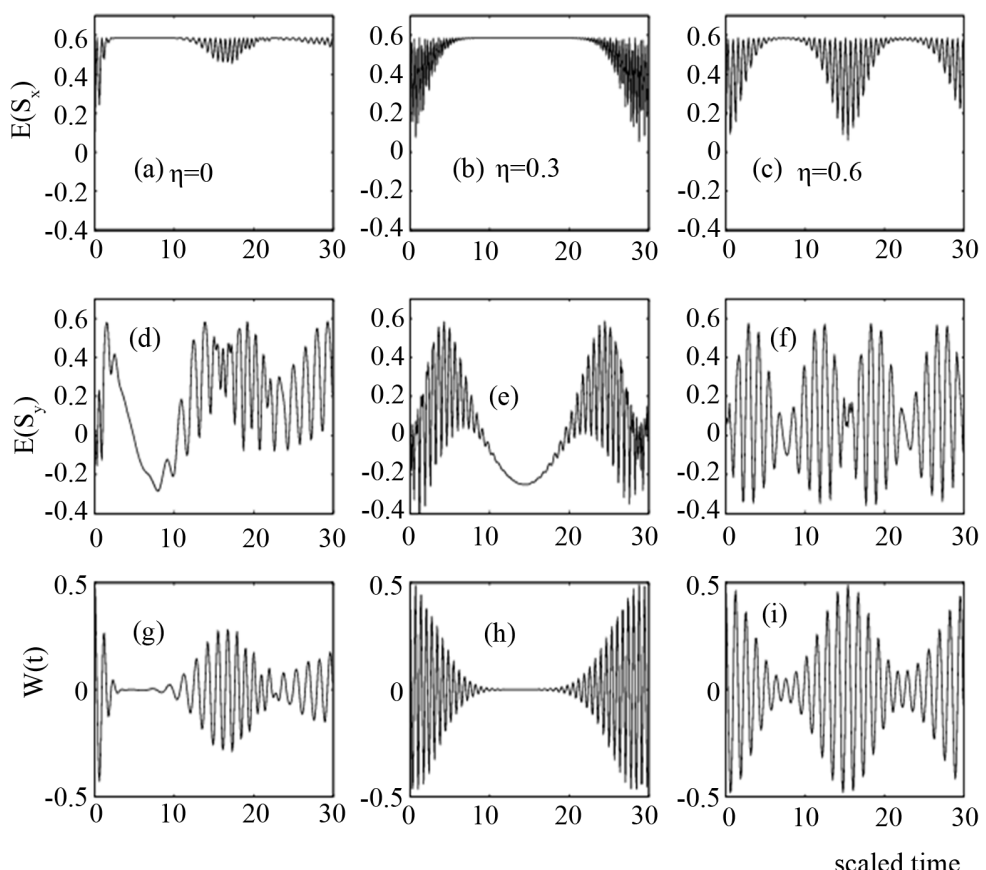

Figure 5. The time evolution of the entropy squeezing factors $E\left(\hat{S}_{x}\right)$, $E\left(\hat{S}_{y}\right)$ and population inversion $W(t)$ of a two-level atom interacting with a single-mode in the excited state $\theta=\varphi=0$, for parameter $\chi=\Delta=0,|\alpha|=6$ and with different values of the Lamb-Dicke parameter $\eta$ where $\eta=0$ in Figures (a,d,g), $\eta=0.5$ in Figures 5(b) (e) (h) and $\eta=0.9$ in Figures 5(c) (f) (i). 
scription of the motion of a trapped ion through Lamb-Dicke parameter $\eta$ and Kerr medium nonlinearity through parameter $\chi$. The first decreases the entropy squeezing but the second increases entropy squeezing.

\section{References}

[1] Shannon, C.E. (1948) A Mathematical Theory of Communication. Bell System Technical Journal, 27, 379-423.

[2] Obada, A.-S.F. and Abdel-Aty, M. (2000) Influence of the Stark Shift and Kerr-Like Medium on the Evolution of Field Entropy and Entanglement in Two-Photon Processes. Acta Physica Polonica, B31, 589.

[3] Steane, A. (1998) Quantum Computing. Reports on Progress in Physics, 61, 117-173. http://dx.doi.org/10.1088/0034-4885/61/2/002

[4] Roy, B. and Roy, P. (2000) New Nonlinear Coherent States and Some of Their Nonclassical Properties. arXiv: quant-ph/0002043.

[5] Fang, M.F., Zhou, P. and Swain, S. (2000) Entropy Squeezing for a Two-Level Atom. Journal of Modern Optics, 47, 1043-1053. http://dx.doi.org/10.1080/09500340008233404

[6] Abdel-Aty, M., Abdel-Khalek, S. and Obada, A.-S.F. (2001) Chaos, Solitons \& Fractals, 12, 2015-2022.

[7] El-Shahat, T.M., Abdel-Khalek, S., Abdel-Aty, M. and Obada, A.-S.F. (2003) Chaos Solitons and Fractals Aspects on Entropy Squeezing of a Two-Level Atom in a Squeezed Vacuum. Journal of Modern Optics, 18, 289-298.

[8] Kuzmich, A., Molmer, K. and Polzik, E.S. (1997) Spin Squeezing in an Ensemble of Atoms Illuminated with Squeezed Light. Physical Review Letters, 79, 4782-4785. http://dx.doi.org/10.1103/PhysRevLett.79.4782

[9] Saito, H. and Ueda, M. (1999) Squeezed Few-Photon States of the Field Generated from Squeezed Atoms. Physical Review A, 59, 3959-3974. http://dx.doi.org/10.1103/PhysRevA.59.3959

[10] Hirschman, L.L. (1957) A Note on Entropy. Journal of Mathematical Physics, 79, 152-156.

[11] El-shahat, T.M., Abdel-Khalek, S., Abdel-Aty, M. and Obada, A.-S.F. (2003) Aspects on Entropy Squeezing of a Two-Level Atom in a Squeezed Vacuum. Chaos, Solitons \& Fractals, 18, 289-298. http://dx.doi.org/10.1016/S0960-0779(02)00652-5

[12] Obada, A.-S.F., Abdel-Khalek, S., Ahmed, M.M.A. and Abo-Kahla, D.A.M. (2009) The Master Equation for a TwoLevel Atom in a Laser Field with Squeezing-Like Terms. Optics Communications, 282, 914-921. http://dx.doi.org/10.1016/j.optcom.2008.10.073

[13] Kares, K. (1983) States, Effects ond Operations. Springer, Berlin. El-Shahat, T.M., Abdel-Khalek, S. and Obada, A.-S.F. (2005) Entropy Squeezing of a Driven Two-Level Atom in a Cavity with Injected Squeezed Vacuum. Chaos, Solitons \& Fractals, 26, 1293-1307. http://dx.doi.org/10.1016/j.chaos.2005.03.013

[14] Matos Filho, R.L. and Vogel, W. (1996) Nonlinear Coherent States. Physical Review A, 54, 4560-4563. http://dx.doi.org/10.1103/PhysRevA.54.4560 